\title{
An EBSPK-/MPPSK-Modem Based Transceiver for Radar-Communications
}

\author{
Yu Yao and Lenan Wu \\ School of Information Science and Engineering, Southeast University, Nanjing 210096, China \\ Correspondence should be addressed to Yu Yao; 1057604987@qq.com
}

Received 1 January 2015; Revised 16 April 2015; Accepted 16 April 2015

Academic Editor: Linda L. Vahala

Copyright (C) 2015 Y. Yao and L. Wu. This is an open access article distributed under the Creative Commons Attribution License, which permits unrestricted use, distribution, and reproduction in any medium, provided the original work is properly cited.

\begin{abstract}
A joint communication and ranging system would constitute a unique platform for future weapon platform or intelligent transportation system to affect the essential tasks of Identification Friend or Foe (IFF) system and location sharing services, in terms of both spectrum efficiency and cost effectiveness. In this paper, the design of efficient modulation system which is suitable for simultaneously performing both data transmission and range measurement is proposed. The approach is based on extended binary phase shift keying (EBPSK) or $M$-ary Position Phase Shift Keying (MPPSK) modulated waveforms utilized in digital communication. In particular, requirements that allow for employing such signals for range measurements are investigated. Also, Constant False Alarm Rate (CFAR) target detection performances of the new proposed system are discussed when target velocity and time delay take different values. Moreover, Doppler tolerance range of impacting filter for demodulating EBPSK/MPPSK pulse signal at $10 \mathrm{GHz}$ is considered. In addition to theoretical considerations, the paper presents simulations and measurement results of the new systems, demonstrating the high spectral utilization of integrated communication and ranging applications.
\end{abstract}

\section{Introduction}

The integration of multiple functions such as radar tasks and communication applications has attracted substantial interest in recent years and sparked a number of research initiatives $[1,2]$. The objective of the joint design is to increase both energy efficiency and spectrum efficiency, and to reduce manufacturing cost. It has the significant military benefits and profound implications for development in technology but is sufficiently challenging.

The AMRFC (Advanced Multifunction Radio Frequency Concept) program is initiated by the office of USA Naval Research. It focuses on a proof-of-principle demonstration of the concept of broadband RF apertures that are capable of simultaneous performance of a large number of radar, electronic warfare, and communication functions from common, low signature apertures. It can use a shared phased array aperture to support communication, radar, and electronic warfare functions [3-5].

An important research area essential for the development of such integrated systems includes the signal design. The design of intelligent waveforms suitable for simultaneously performing both data transmission and radar sensing has been proposed in some literature [6-9].

The literature [10] presents a novel method for multiplying the binary phase shift keying (BPSK) communication signals based on direct sequence spread spectrum (DSSS) by linear frequency modulation (LFM) radar signals. It means that two signals are allowed to bidirectionally transmit message on public channels simultaneously. After linear and homomorphic filtering, multiplicative echo signal could be transformed into additive signals at the receiver. Radar echo and communication signal could be separated by using blind source separation. Unfortunately, one of the main drawbacks of this method will damage the ranging performance, especially for the detection of small target.

However, a novel waveform is the efficient modulated signal [11] which is a well-known candidate for joint radarcommunication (JRC); the work reported in [12] investigated the integrated system based on pseudorandom (PN) sequence. Meanwhile, the receiver of the system equips a much narrower pass-band filter named digital impacting filter (DIF) $[13,14]$; it can extract the tiny phase modulation to ease demodulation. The previously proposed JRC in 
the literature [15] performs digital communications and radar measurements periodically. In this paper, the new integrated system with impacting filter and matched filter is proposed, which can simultaneously operate digital communications and radar applications.

The rest of this paper is organized as follows. Section 2 introduces EBPSK and MPPSK modulated waveform characters. Section 3 described the correlation processing for performing the radar measurement with the coded signal. Section 4 illustrates block diagrams of the proposed integrated system which are partly described. In Section 5, the Constant False Alarm Rate (CFAR) target detection performance of the proposed system is deduced. Some indicative simulation results and performance analysis are presented in Section 6. And, finally, Section 7 gives the conclusion of the paper.

\section{EBPSK/MPPSK Modulated Waveforms}

EBSPK-/MPPSK-modem based JRC transceiver transmits EBPSK/MPPSK pulse signals, which is efficient regarding the amount of information they convey on the bandwidth they occupied. The modulated extended binary phase shift keying (EBPSK) pulse waveforms are defined in [11]. Consider

$$
\begin{aligned}
& g_{0}(t)=A \sin 2 \pi f_{c} t, \quad 0 \leq t<N T_{c}, \\
& g_{1}(t)= \begin{cases}-B \sin 2 \pi f_{c} t, & 0 \leq t<K T_{c}, \\
A \sin 2 \pi f_{c} t, & K T_{c} \leq t<N T_{c}\end{cases}
\end{aligned}
$$

with $g_{0}(t)$ and $g_{1}(t)$ being modulation waveforms of symbols " 0 " and " 1 " and $f_{c}$ and $T_{c}$ representing the carrier frequency and the carrier period, respectively. $K$ and $N$ stand for the number of the carrier periods in each time slot and the number of the carrier periods in each symbol, respectively. $K$ : $N$ denotes modulation duty cycle. And three kinds of simplified expressions are presented in the paper as follows.

(1) For $B=A$, the EBPSK pulse waveforms are defined as Phase Reversal Modulation (PRM) waveforms:

$$
\begin{aligned}
& g_{0}(t)=A \sin 2 \pi f_{c} t, \quad 0 \leq t<N T_{c}, \\
& g_{1}(t)= \begin{cases}-A \sin 2 \pi f_{c} t, & 0 \leq t<K T_{c}, \\
A \sin 2 \pi f_{c} t, & K T_{c} \leq t<N T_{c} .\end{cases}
\end{aligned}
$$

(2) For $A=0$, the EBPSK pulse waveforms are defined as Missing Cycle Modulation (MCM) waveforms:

$$
\begin{aligned}
& g_{0}(t)=A \sin 2 \pi f_{c} t, \quad 0 \leq t<N T_{c}, \\
& g_{1}(t)= \begin{cases}0, & 0 \leq t<K T_{c}, \\
A \sin 2 \pi f_{c} t, & K T_{c} \leq t<N T_{c} .\end{cases}
\end{aligned}
$$

(3) For $B=0$, the EBPSK pulse waveforms are essentially equivalent to the traditional pulse radar waveforms, which are expressed as

$$
\begin{aligned}
& g_{0}(t)=0, \quad 0 \leq t<N T_{c}, \\
& g_{1}(t)= \begin{cases}-B \sin 2 \pi f_{c} t, & 0 \leq t<K T_{c}, \\
0, & K T_{c} \leq t<N T_{c} .\end{cases}
\end{aligned}
$$

In the following, the MPPSK modulated pulse waveforms are defined by [15], and the simplified expression is presented in the paper as follows:

$$
\begin{aligned}
& s_{1}(t) \\
& =\left\{\begin{array}{lll}
\sin \left(2 \pi f_{c} t\right) & & 0 \leq t<N T_{c}, k=0 \\
\sin \left(2 \pi f_{c} t\right) & 0 \leq t \leq(k-1) K T_{c} & \\
-\sin \left(2 \pi f_{c} t\right) & (k-1) K T_{c}<t<k K T_{c} & 1 \leq k \leq M-1 \\
\sin \left(2 \pi f_{c} t\right) & k K T_{c} \leq t<N T_{c}, &
\end{array}\right.
\end{aligned}
$$

where $m(m=0,1, \ldots, M-1)$ are $M$-ary $(M \geq 2)$ source symbols. From (4), traditional pulse radar waveform is a special case of EBPSK/MPPSK modulated waveform.

\section{Signal Coding}

The baseband expression of the EBPSK/MPPSK composite signal is

$$
s_{E}(t)=\sum_{i=0}^{L N N_{\mathrm{sym}}-1} a(i) g\left(t-i T_{c}\right)
$$

With $N_{\text {sym }}$ being the number of modulation symbols composing the signal frame, $L$ is the code sequence. $T_{c}=1 / f_{c}$ denotes the symbol duration, $g(t)$ is the elementary baseband pulse shape, and $a(i)$ represents the discrete amplitude and phase states obtained through EBPSK or MPPSK modulation scheme.

The correlation processing for performing the radar measurement with the reflected signal is addressed in detail. If the transmitted signal is scattered back from an object located in the range $R$, the echo signal $r_{E}(t)$ can be expressed as

$$
\begin{aligned}
r_{E}(t) & =A s_{E}^{\prime}\left(t-\frac{2 R}{c}\right) \\
& =A \sum_{i=0}^{L N N_{\text {sym }}-1} c(i) g\left(t-\frac{2 R}{c}-i T\right)
\end{aligned}
$$

with the amplitude factor $A$ describing the attenuation and phase shift occurring due to the scattering process and $c$ being the speed of light. The radar processor estimates the range to the scattering object by calculating the cross-correlation 


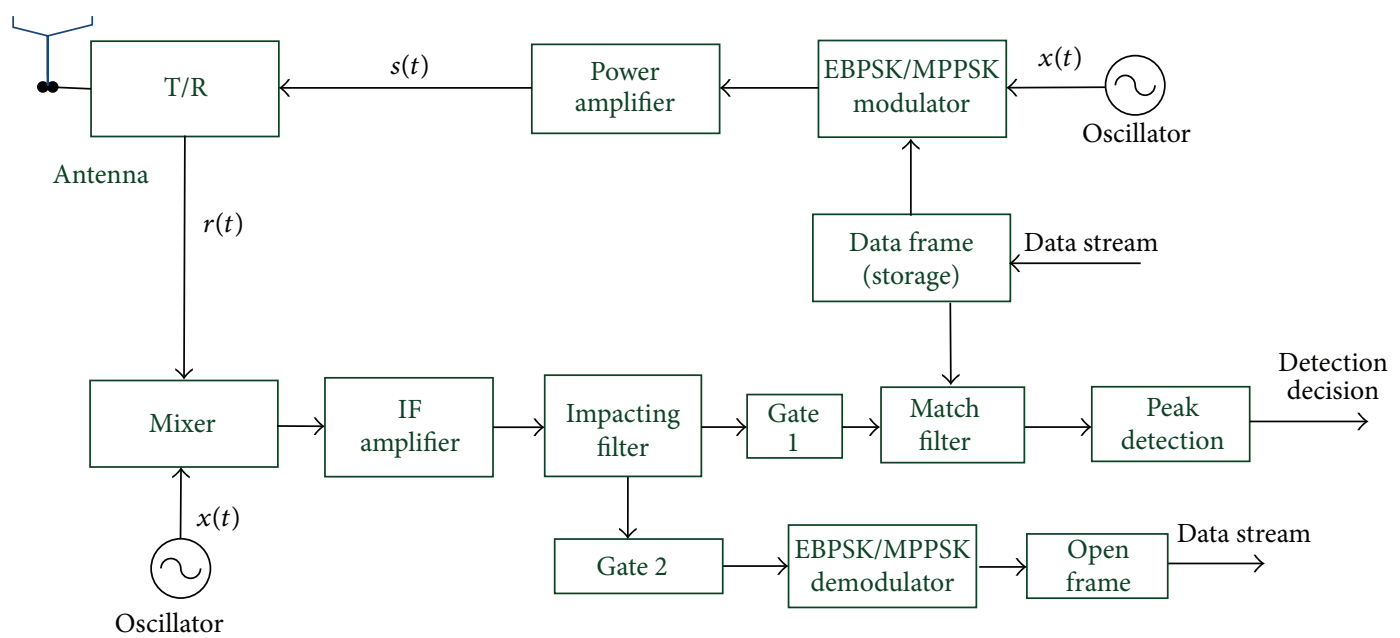

FIGURE 1: EBSPK-/MPPSK-modem based radar-communication transceiver.

function, $r_{y x}(t, \tau)$, between the received and the transmitted signals. Consider

$$
\begin{aligned}
& r_{r s}(t, \tau)=A \sum_{i=0}^{L N N_{\text {sym }}-1} \sum_{k=0}^{L N N_{\text {sym }}-1} c(i) c(k) \\
& \cdot \int_{t=0}^{L N N_{\text {sym }} T} g\left(t-\frac{2 R}{c}-i T\right) g(t-\tau-k T) d t .
\end{aligned}
$$

Obviously, the integral in (9) describes the autocorrelation function $r_{g g}(\tau)$ of the baseband pulse shape $g(t)$ shifted in time:

$$
\begin{gathered}
r_{r s}(\tau)=A \sum_{i=0}^{L N N_{\text {sym }}-1} \sum_{k=0}^{L N N_{\text {sym }}-1} c(i) c(k) \\
\cdot r_{g g}\left(\tau-\frac{2 R}{c}+(k-i) T\right) .
\end{gathered}
$$

The properties of the code sequence have a direct impact on the result of the correlation performed in the receiver. Then, $r_{g g}(\tau)$ will show a peak value under the condition $\tau=0$. In order that the time shifted autocorrelation function in (7) contributes its maximum value, the following condition must hold:

$$
\tau=\frac{2 R}{c}-(k-i) T
$$

For $\tau=0$,

$$
k=i+\left\lfloor\frac{2 R}{c T}\right\rfloor .
$$

Hence, for an index shift determined by the distance of the object a main peak appears in $r_{g g}(\tau)$.

\section{The Principle of the Integrated System}

4.1. EBPSK/MPPSK Transmitter. The main working processes of pulse signals transmitter based EBPSK/MPPSK modulation are described as follows.
Communication data stream should be scrambled by pseudorandom $(\mathrm{PN})$ sequence to make sure that the data symbols "1" and "0" appear to be equiprobability. Transmitter equipment is mainly composed of pulse train EBPSK/MPPSK modulation generators, as shown in Figure 1. The scrambled data stream would be truncated to a communication data frame on a radar pulse width $\tau$ cycle. The data frames are modulated by the coded EBPSK/MPPSK pulse modulator defined by formulas (1) and (5).

Transmitter sends EBPSK/MPPSK modulated pulses frame by frame on a radar pulse repetition period $T$ cycle. And communication data with bit period $T_{1}$ in a pulse width $\tau$ would be defined as radar subpulse repetition period.

After power amplification in the transmitter, the coded EBPSK/MPPSK modulated pulses are sent into the air by antenna within the emission timeslot of transmitter/receiver switch. Current data frame is always saved in the storage. Follow-up of data frame will be updated on a radar pulse repetition period $T$ cycle.

4.2. EBPSK/MPPSK Receiver. The reception equipment is made by two kinds of receivers: one is mainly composed of matched filter, and the other is mainly composed of EBPSK/MPPSK demodulator as shown in Figure 1. The principle of EBPSK/MPPSK demodulation had been described in literature [13]. And the main working processes of EBPSK/ MPPSK modulated pulses receiver based on the matching process and the impacting process are described as follows.

The antenna receives radar echoes and communication signals sent by the target; then the received signals are amplified by preamplifier at the receiver. The raw amplified signals are extracted by downconversion from RF to IF.

Radar-communication mixed signals are split into two channels by using gate 1 and gate 2 . Two gates are of the same time width but with different opening time. The opening times of two gates are set as pulse width $\tau$. The opening time of gate 2 is relatively lagging behind gate 1 , and the delay time is equal to pulse width $\tau$. 


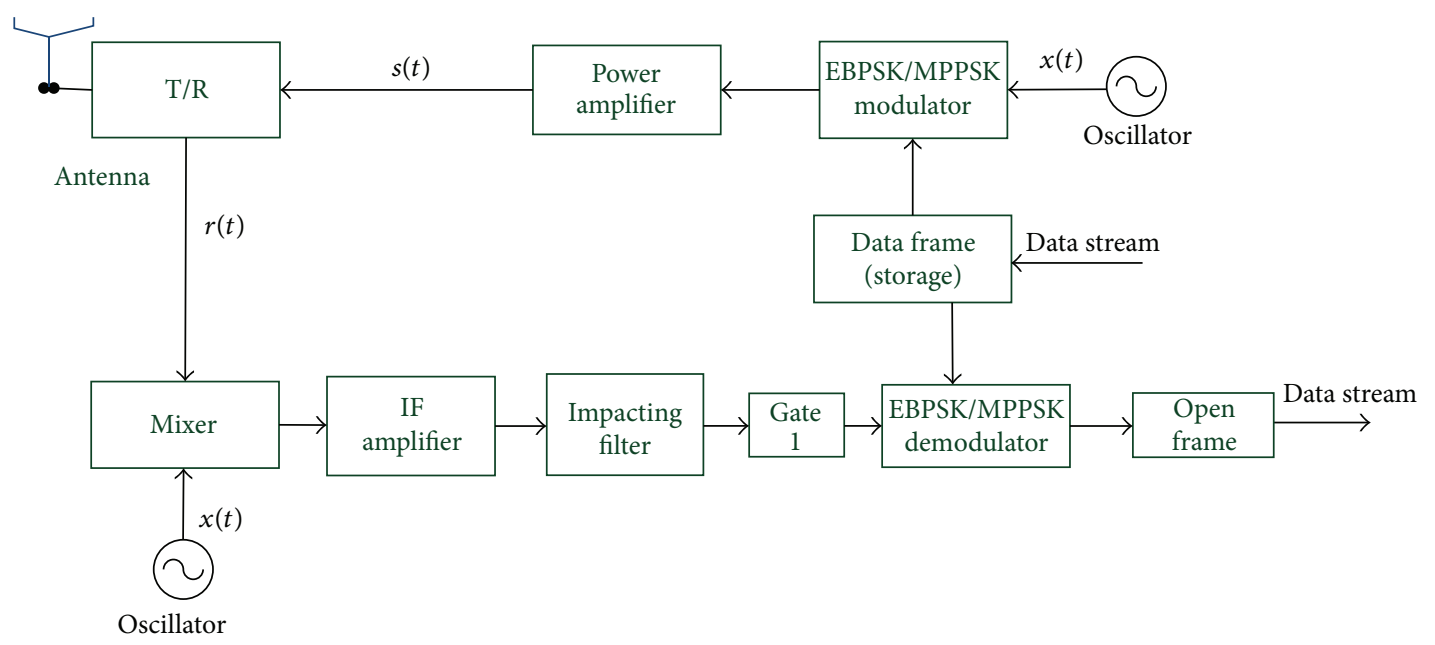

FIGURE 2: EBSPK-/MPPSK-modem based radar-communication transceiver.

4.3. Echo Signal Detection. EBSPK-/MPPSK-modem based radar-communication transceiver transmits composite pulse signals for the ranging function and communication operation. Pulse repetition period is $T$ and pulse width is $\tau$.

Radar wave will be immediately reflected when it reaches metal shell of target. The process of reflection will spend $\tau$ in theory. From Figure 1, gate 1 is used in the proposed burst transmission system for selecting radar signals and isolating communication date sent by other systems from target vehicle. Radar echoes from the gate 1 are sent into match filter for correlation processing with copy of current frame data stored in data frame storage. A simple peak detection and threshold decision can perform the demodulation for the output of the correlation operation, which results in the simple receiver structure. The distance of the target relative to the radar would be estimated by measuring time delay of peak of the correlation function. Constant False Alarm Rate (CFAR) moving target detector for High Resolution Radar (HRR) is used in the proposed system. After the matched filtering, current communication data would be cleared in the frame memory, and the next frame will be stored.
Obviously, the proposed systems of both parties would send the communication date frame after processing a data frame at the receiving end. The minimum time of processing a data frame is radar pulse width $\tau$. Therefore, the interference from strong communication signals would be easily avoided by using time division scheme.

4.4. EBPSK/MPPSK Demodulation. Gating time of gate 2, which is pulse width $\tau$, lags behind gate 1 for pulse width $\tau$. So the identification and demodulation of EBPSK/MPPSK communication signals would not be affected by radar echo signals reflected by close-in target. Noise and interference of communication signal would be easily removed in the time domain, because date frames are demodulated and processed on a pulse width cycle.

The engineering implement of processing EBPSK/ MPPSK modulated communication data by the impacting filtering is given in [12]. In fact, impacting filter (IF) is a special match filter for processing phase modulation signals. It simplifies the structure of the receiver greatly, because the order of digital infinite impulse response (IIR) filter is low. In this paper, the expression and related parameter of the IF are

$$
H(z)=\frac{b_{0}+b_{1} \cdot z^{-1}+b_{2} \cdot z^{-2}}{1-a_{1} \cdot z^{-1}-a_{2} \cdot z^{-2}-a_{3} \cdot z^{-3}-a_{4} \cdot z^{-4}-a_{4} \cdot z^{-5}-a_{4} \cdot z^{-6}-a_{4} \cdot z^{-7}-a_{4} \cdot z^{-8}} .
$$

The zero parameters of the IF are selected as $b_{0}=b_{2}=1$ and $b_{1}=-1.61818507230537$, and the pole parameters of the IF in this paper are selected as follows:

$$
\begin{aligned}
& a_{1}=-6.08241620884024 ; a_{2}=17.4061856872407 \\
& a_{3}=-30.1745102291534 ; a_{4}=34.5134024239294 \\
& a_{5}=-26.6242346202708 ; a_{6}=13.55133760021 \\
& a_{7}=-4.17834684730643 ; a_{8}=0.606151111558408
\end{aligned}
$$

When EBPSK/MMPSK modulated signals pass the impacting filter, the special impacting filter can transform the tiny waveform difference into amplitude impacting, which is relatively stable within certain Doppler frequency shift limits.

When mobile platform does not need to operate ranging function, the proposed integrated system can be simplified. As shown in Figure 2, the EBPSK/MPPSK modulated signal is only demodulated by EBPSK/MPPSK demodulator. In 


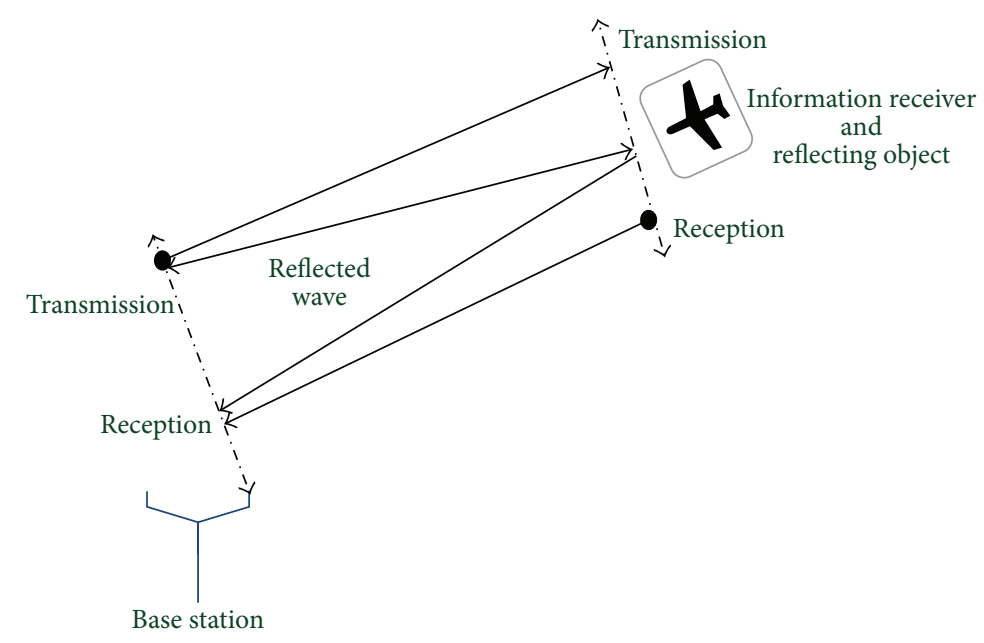

FIgURE 3: The schematic of communication and ranging process.

fact, the system is a highly efficient modulation digital communication system.

Different from traditional radar stations, the proposed design scheme can be applied to the ground-based radar station and mobile platform. The ground-based radar station sends the instruction or large amounts of data to mobile platform by using the EBPSK/MPPSK modulated signals. Then the transmitted signal is scattered back from a mobile platform located in the range $R$. The received signal can be demodulated by matched filter at the receiver, which would produce high accurate measurement of moving target's range and velocity. At the same time, the ground-based radar station equipped with a new proposed system can demodulate EBPSK/MPPSK signals sent by mobile platform. Impacting filter at the receiver is made to realize full-duplex communications. The scheme would also be available to detection and communication between two mobile platforms. Figure 3 shows the sketch of this process.

\section{Target Detection}

5.1. Target Velocity and Range Both Are Known. CFAR target detection performance of the proposed system is deduced when the target velocity and range both are known. CFAR target detection algorithm could be written as

$$
\gamma=\mathbf{r}^{H} \mathbf{s} \underset{H_{0}}{\stackrel{H_{1}}{\gtrless}} \gamma_{0} .
$$

With $\mathbf{r}$ representing discrete sampled echo signals, $\mathbf{s} \epsilon$ $\mathbb{C}^{N \times 1}$ describes discrete sampled sending signals, $\gamma_{0}$ is CFAR detection threshold, and target echo signals would be written as

$$
\mathbf{r}=\mathbf{s}+\mathbf{n}
$$

where $\mathbf{s}=\mathbf{s}_{E}, \mathbf{s}=\mathbf{s}_{M}$ stand for EBPSK or MPPSK pulse sending signals, separately. The pulse echo is disturbed by
Additive-White Gaussian Noise (AWGN). False alarm probability can be expressed as

$$
\begin{aligned}
P_{\mathrm{fa}} & =P\left(H_{1} \mid H_{0}\right)=\int_{\gamma_{0}}^{\infty} \frac{1}{\sqrt{2 \pi} \sigma} \exp \left(-\frac{\gamma^{2}}{2 \sigma^{2}\|\mathbf{s}\|_{2}^{2}}\right) d \gamma \\
& =Q\left(\frac{\gamma_{0}}{\sigma\|\mathbf{s}\|_{2}}\right),
\end{aligned}
$$

where $(x)=\int_{x}^{\infty}(1 / \sqrt{2 \pi}) \exp \left(-t^{2} / 2\right) d t$ and the target detection threshold can be deduced as

$$
\gamma_{0}=Q^{-1}\left(P_{\mathrm{fa}}\right) \sigma\|\mathbf{s}\|_{2} .
$$

The corresponding target detection probability would be written as

$$
\begin{aligned}
P_{d} & =P\left(H_{1} \mid H_{1}\right) \\
& =\int_{\gamma_{0}}^{\infty} \frac{1}{\sqrt{2 \pi} \sigma} \exp \left(-\frac{\left(\gamma-\|\mathbf{s}\|_{2}^{2}\right)^{2}}{2 \sigma^{2}\|\mathbf{s}\|_{2}^{2}}\right) d \gamma \\
& =Q\left(\frac{\gamma_{0}-\|\mathbf{s}\|_{2}^{2}}{\sigma\|\mathbf{s}\|_{2}}\right) .
\end{aligned}
$$

According to the definition,

$$
\mathrm{SNR}=\frac{\|\mathbf{s}\|_{2}^{2}}{N \cdot \sigma^{2}} .
$$

The corresponding target detection probability would be written as

$$
P_{d}=Q\left(Q^{-1}\left(P_{\mathrm{fa}}\right)-\sqrt{\mathrm{SNR} \cdot N}\right) .
$$

5.2. Unknown Target Velocity. When the target velocity is unknown, it does not need to estimate carrier frequency offset caused by target's movement. According to the CFAR target detection algorithm, target echo signals would be written as

$$
\mathbf{y}=\mathbf{s}_{D}+\mathbf{n},
$$


where $\mathbf{s}_{D}$ is Doppler frequency offset signal. The corresponding target detection probability would be written as

$$
\begin{aligned}
P_{d} & =Q\left(Q^{-1}\left(P_{\mathrm{fa}}\right)-\frac{\mathbf{s}^{H} \mathbf{s}_{D}}{\sigma\|\mathbf{s}\|_{2}}\right) \\
& =Q\left(Q^{-1}\left(P_{\mathrm{fa}}\right)-\frac{\mathbf{s}^{H} \mathbf{s}_{D} \sqrt{N \cdot \mathrm{SNR}}}{\|\mathbf{s}\|_{2}^{2}}\right) .
\end{aligned}
$$

5.3. Unknown Target Range. When the target range is unknown, target echo signals would be written as

$$
\mathbf{y}=\mathbf{s}_{R}+\mathbf{n}
$$

with $\mathbf{s}_{R}$ standing for pulse signal with time delay; CFAR target detection probability is

$$
\begin{aligned}
P_{d} & =Q\left(Q^{-1}\left(P_{\mathrm{fa}}\right)-\frac{\mathbf{s}^{H} \mathbf{s}_{R}}{\sigma\|\mathbf{s}\|_{2}}\right) \\
& =Q\left(Q^{-1}\left(P_{\mathrm{fa}}\right)-\frac{\mathbf{s}^{H} \mathbf{s}_{R} \sqrt{N \cdot \mathrm{SNR}}}{\|\mathbf{s}\|_{2}^{2}}\right) .
\end{aligned}
$$

\section{Simulation Results}

\subsection{Setting the Parameters}

6.1.1. Transmitter. In transmitter, LFM pulse and EBPSK/ MPPSK pulse on the carrier frequency $f_{c}=10 \mathrm{GHz}$ are modulated by using the proposed modulator. Sampling frequency $f_{s}$ is $100 \mathrm{GHz}$. The bandwidths of these signals are $B=$ $50 \mathrm{MHz}$ and pulse durations of these signals are $\tau=20 \mu \mathrm{s}$. Pulse repetition period $T=200 \mu \mathrm{s}$ (PRF is $5 \mathrm{kHz}$ ).

6.1.2. Receiver. Radar echo signals on the carrier frequency $f_{c}=10 \mathrm{GHz}$ received by the antenna downconvert to IF $=100 \mathrm{MHz}$ for the back-end to process. And the sampling frequency $f_{s}$ is $1 \mathrm{GHz}$. Impacting filter given in this paper is an IIR digital filter as shown in Figure 4. The design principle of parameters given in (12) is to make sure that IF signal is in the center of the amplitude-frequency curve slope of impacting filter. Setting EBPSK/MPPSK modulation parameter $K=2$ is to assure that the bandwidth of EBPSK/MPPSK signal $B$ is also equal to $50 \mathrm{MHz}$, the same as LFM signal. If transmitter sends LFM signal, the echo signal would be demodulated and detected by receiver only using the matched filter.

6.1.3. Application Scenario and Environment. Taking certain helicopter as example, the maximum operating range of JRC transceiver is $35 \mathrm{~km}$. We consider the detection performance of the proposed JRC system in AWGN channel.

6.2. The Improved Amounts of Output SNR. Radar application principle of the proposed JRC is to produce a pseudorandom bit stream in data frame storage, which can be reused in applications. Using the quasi-periodicity and distribution, data stream can be modified and the encryption can be achieved. The proposed system is equivalent to the pulse

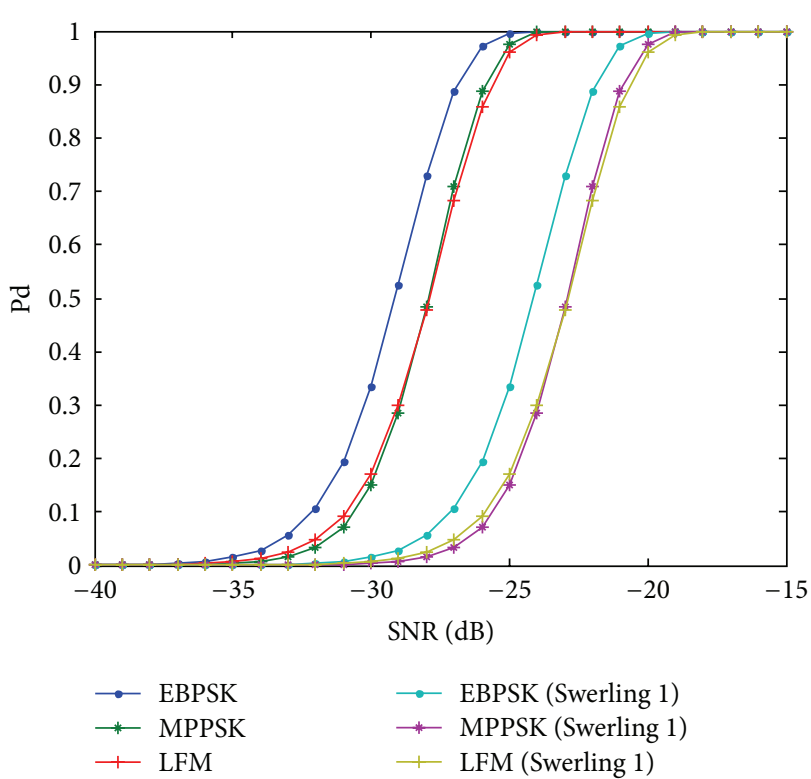

FIGURE 4: CFAR target detection probability comparisons for $v=$ $400 \mathrm{~m} / \mathrm{s}$.

compression system with phase-coded signals. The ratio of Doppler frequency shift to carrier frequency $f_{d} / f_{c}$ is related to carrier frequency and has nothing to do with IF.

For EBPSK modulated signal, setting modulation parameter $N=8,7$, and 6; 1024 bit $\mathrm{M}$ sequences code and input SNR $=1 \mathrm{~dB}$; the improved amounts of out SNR of LFM and EBPSK modulation radar systems in different target velocity and Doppler frequency shift are provided in Table 1.

The simulation results of Table 1 illustrate the improved amounts of output SNR in different systems.

Aiming at the low speed target detection (below $750 \mathrm{~m} / \mathrm{s}$ ), the SNR improvement of the proposed system with EBPSK-/ MPPSK-modem may be reduced by approximately $0.5 \mathrm{~dB}$ as compared with the performance of traditional system with LFM and coherent demodulation, but the difference of the SNR improved amount decreased gradually with the target velocity increased. The SNR performances of the EBPSK/MPPSK JRC and traditional LFM radar system just have less than $0.2 \mathrm{~dB}$ difference at $750 \mathrm{~m} / \mathrm{s}$. The result of the proposed JRC would be better when the velocity of moving target is higher than $750 \mathrm{~m} / \mathrm{s}$, which is caused by impacting filter method and the selection of modulation parameters. When the velocity of moving target increases to 10 times the speed of sound (about $3400 \mathrm{~m} / \mathrm{s}$ ), the SNR improvement of the proposed system would be increased by approximately $1.5 \mathrm{~dB}$ as compared with that of the LFM radar system. Therefore, comparing with LFM radar system, the EBPSK/MPPSK JRC has better performance of detection to high speed target.

It is noteworthy that the proposed system shows the robustness with regard to the velocity of the moving target.

6.3. CFAR Target Detection Performance. In this section, the performance of the proposed EBPSK/MPPSK system is 
TABLE 1: Comparative simulation on the improved amounts of output SNR of LFM and EBPSK modulation radar systems.

\begin{tabular}{|c|c|c|c|c|c|c|}
\hline \multirow{3}{*}{ Target velocity $(\mathrm{m} / \mathrm{s})$} & \multicolumn{2}{|c|}{ Doppler shift } & \multicolumn{4}{|c|}{ The improved amounts of output SNR of these sending signals $(\mathrm{dB})$} \\
\hline & \multirow{2}{*}{$\begin{array}{c}\text { Absolute value } \\
\qquad(\mathrm{KHz})\end{array}$} & \multirow{2}{*}{$\begin{array}{l}\text { Relative value } \\
\qquad\left(\times 10^{-6}\right)\end{array}$} & \multirow{2}{*}{ LFM } & \multicolumn{3}{|c|}{ EBPSK coded modulation } \\
\hline & & & & $N=8$ & $N=7$ & $N=6$ \\
\hline 0 & 0 & 0 & 23.4 & 22.8 & 22.8 & 23 \\
\hline 100 & 0.67 & 0.067 & 23.2 & 22.6 & 22.7 & 22.8 \\
\hline 200 & 1.3 & 0.13 & 23.1 & 22.6 & 22.7 & 22.7 \\
\hline 400 & 2.7 & 0.27 & 23.2 & 22.7 & 22.8 & 22.8 \\
\hline 750 & 5 & 0.5 & 22.6 & 22.6 & 22.6 & 22.8 \\
\hline 1400 & 9.3 & 0.93 & 22.3 & 22.4 & 22.5 & 22.5 \\
\hline 2000 & 13 & 1.3 & 22 & 22.5 & 22.6 & 22.6 \\
\hline 2500 & 16.7 & 1.7 & 21.5 & 22.4 & 22.4 & 22.5 \\
\hline 2800 & 18.7 & 1.9 & 21 & 22.5 & 22.6 & 22.7 \\
\hline 3000 & 20 & 2.0 & 21 & 22.2 & 22.3 & 22.5 \\
\hline 3400 & 22.7 & 2.3 & 20.6 & 22.1 & 22.2 & 22.1 \\
\hline
\end{tabular}

simulated. A summary of the most important parameters of the simulation model is provided as follows.

\section{Parameter Value}

Carrier frequency: $f_{1}=10 \mathrm{GHz}$.

EBPSK modulation parameters: $N=7$.

MPPSK modulation parameters: $N=7, M=3$.

False alarm probability: $P_{\alpha}=0.05 \%$.

Target detection probability: $P_{d}=0.9$.

Target velocity range: $0 \mathrm{~m} / \mathrm{s} \sim 3400 \mathrm{~m} / \mathrm{s}$.

In the following simulations, the relations between CFAR target detection probability with the SNR are shown in Figures 5, 6, and 7 for pulse width $T=200 \mu$ s and pulse accumulation number $N=10$. The results are calculated by (20) and obtained from 3000 Monte Carlo simulations under different SNRs.

Figure 4 shows CFAR target detection probability comparisons after LFM and EBPSK/MPPSK echo signals have been successfully compressed at the receiving end. As shown in Figure 4, the simulation shows that, at the velocity $v=$ $400 \mathrm{~m} / \mathrm{s}$, for the nonfluctuation targets, the SNR performance of EBPSK system would be improved by approximately $2.1 \mathrm{~dB}$ as compared with LFM system. The result of MPPSK system is slightly $0.2 \mathrm{~dB}$ better than LFM system.

For the fluctuation targets (Swerling models 1), the SNR performance of EBPSK system would be improved by approximately $2.5 \mathrm{~dB}$ as compared with that of the LFM system. The result of MPPSK system is slightly $0.3 \mathrm{~dB}$ better than LFM system.

As depicted in Figure 5, it illustrates the nonfluctuation and fluctuation target detections; the simulation shows that, at the target velocity $v=750 \mathrm{~m} / \mathrm{s}$, for the nonfluctuation targets, the SNR performance of EBPSK system would be improved by approximately $2.5 \mathrm{~dB}$ as compared with LFM system, while the result of MPPSK system is slightly better than LFM system by $0.4 \mathrm{~dB}$.

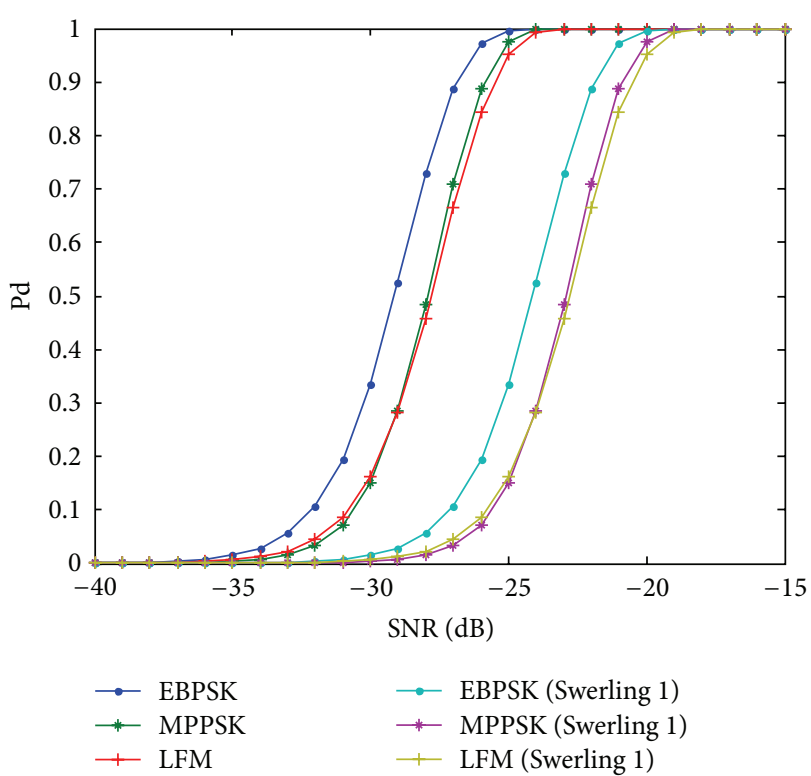

FIGURE 5: CFAR target detection probability comparisons for $v=$ $750 \mathrm{~m} / \mathrm{s}$

For fluctuation targets (Swerling models 1), the SNR performance of EBPSK system would be improved by approximately $2.3 \mathrm{~dB}$ as compared with that of the LFM system, while the result of MPPSK system is slightly better than that of the LFM system by $0.5 \mathrm{~dB}$.

As given in Figure 6, the simulation shows that, at the target velocity $v=2000 \mathrm{~m} / \mathrm{s}$, for the nonfluctuation targets, the SNR performance of EBPSK system would be improved by approximately $3 \mathrm{~dB}$ as compared with that of the LFM system, while the result of MPPSK system is $1 \mathrm{~dB}$ better than that of the LFM system.

For fluctuation targets (Swerling models 1), the EBPSK system would gain about $3.2 \mathrm{~dB}$ in SNR performance as compared with the LFM system, and the MPPSK system is slightly better than LFM system by $1 \mathrm{~dB}$. 


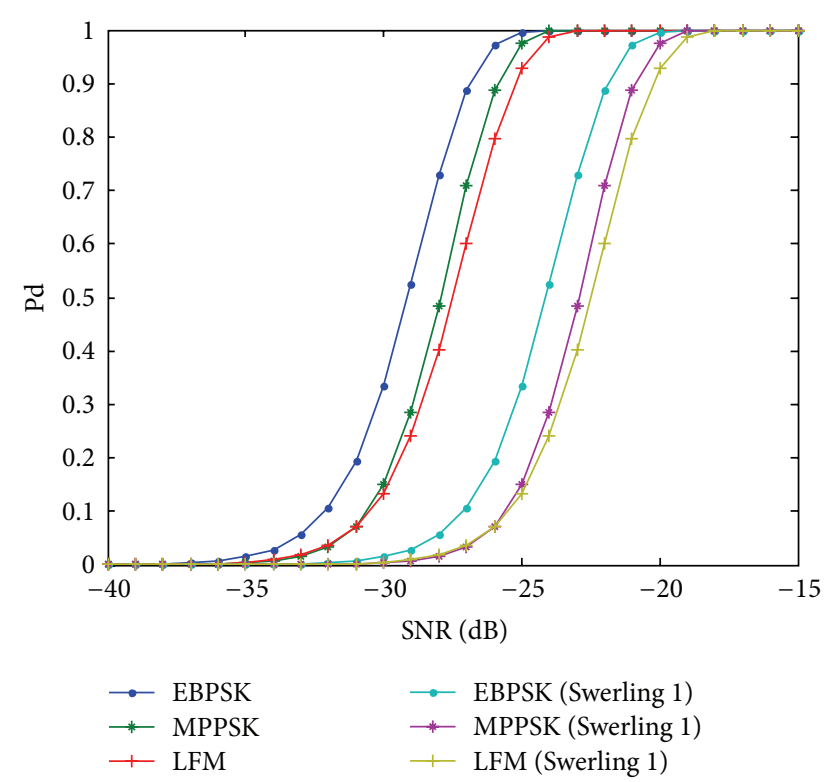

FIGURE 6: CFAR target detection probability comparisons for $v=$ $2000 \mathrm{~m} / \mathrm{s}$.

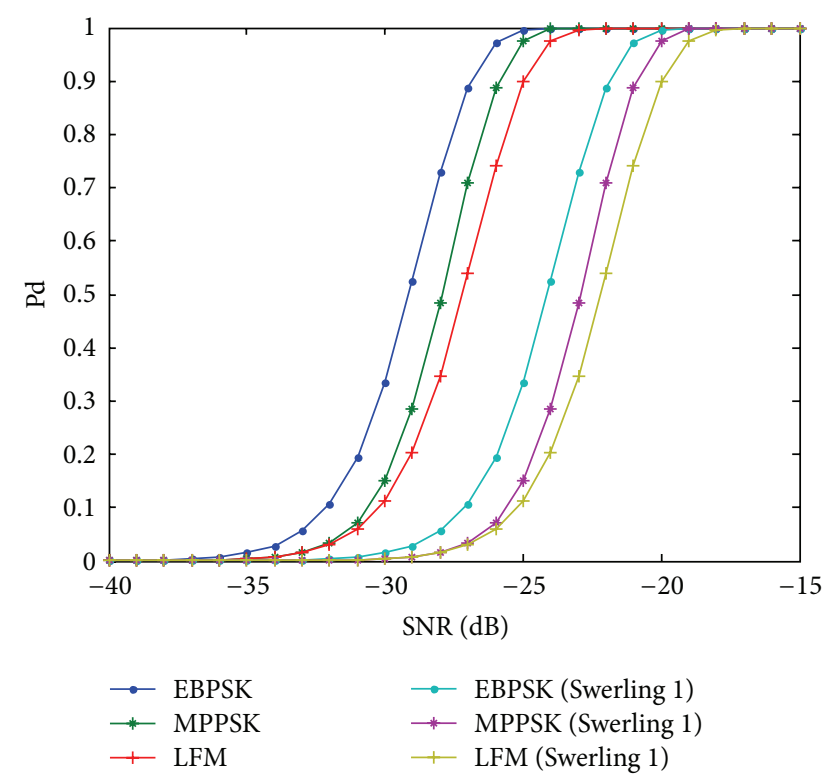

FIGURE 7: CFAR target detection probability comparisons for $v=$ $3400 \mathrm{~m} / \mathrm{s}$.

As shown in Figure 7, the SNR performance demonstrates that, at the target velocity $v=3400 \mathrm{~m} / \mathrm{s}$, for the nonfluctuation targets, the EBPSK system would be $3.5 \mathrm{~dB}$ better than LFM system, and the MPPSK system is about $1.4 \mathrm{~dB}$ better than LFM system.

For fluctuation targets (Swerling models 1), the EBPSK system would be $3.5 \mathrm{~dB}$ better than LFM system, and the MPPSK system is $1.5 \mathrm{~dB}$ better than LFM system. Thus it can be seen that the detection performance of the proposed EBSPK-modem system increased with the target velocity increased. Simultaneously, research on the optimal modem method is underway; the system performance still has room for improvement.

6.4. Data Communication Operation. For data communication operation, the real random sending data will be stored in the frame memory. From (1) and (5), the transmission rate of EBPSK/MPPSK modulated signal is proportional to carrier frequency $f_{c}$, but inversely proportional to modulation parameter $N$.

The primary function of the JRC receiver is to amplify echo signals coming from the antenna and downconvert its frequency to IF $=100 \mathrm{MHz}$ for followed procession. So the radar parameters shall be calculated according to IF = $100 \mathrm{MHz}$. Setting modulation parameter $N=8$, we can get the following parameters.

(i) For EBPSK modulated pulse, burst transmission bit rate of the proposed system is $100 / 8=12.5 \mathrm{Mbps}$; since the duty ratio of the radar periodic pulse signal is $20 / 200$, equivalent continuous transmission bit rate is $1.25 \mathrm{Mbps}$.

(ii) For MPPSK modulated pulse signal and modulation parameters $M=4$, burst transmission bit rate and equivalent continuous transmission bit rate of the proposed JRC are $2 * 100 / 8=25 \mathrm{Mbps}$ and $2.5 \mathrm{Mbps}$, respectively.

This means that, compared with EBPSK modulation, a longer length of PN code could be achieved by using MPPSK modulation after phase-coded pulse compression for sending signals. The proposed JRC system using MPPSK modulation instead of EBPSK modulation would get higher range resolution, better ranging accuracy, and more communication data. According to the relational expression,

Data volume of a beam
$\quad=$ Bit rate $\times$ Pulse width $\times$ Pulse number.

If the proposed system would transmit-receive 20 pulses within a radar beam, $20 * 2000 / 8=5 \mathrm{kB}$ and $20 * 4000 / 8=$ $10 \mathrm{kB}$ per beam would be transmitted by the proposed system based EBPSK and MPPSK modulation, respectively. When the communication target is locked by radar beam, both the sender and receiver agree that they are ready to carry out continuous reliable communication with rate $1.25 \mathrm{Mbps}$ or $2.5 \mathrm{Mbps}$. For data communication operation, transmission bit rate of the proposed JRC based on MPPSK modulation would be twice as high as that of on EBPSK modulation at least.

\section{Conclusions}

A novel JRC concept for the EBSPK-/MPPSK-modem based transceiver has been presented. The proposed joint radarcommunication system is composed primarily of EBPSK/ MPPSK signals generator and integrated receiver architecture, which is illustrated in block diagram; matched and impacting filter that essentially determine EBPSK/MPPSK signal demodulation are emphasized in the paper. 
For the ranging function, CFAR detection performance with MPPSK modulated pulse signals is a little worse than that with EBPSK modulated pulse signals, but the range resolution with MPPSK signal is higher. The spectral efficiency and the data rate in communication mode are also better. Two kinds of signals would be selected to satisfy the different requirements of radar ranging and data communication. With simulations the operability of the proposed JRC concept and its superior performance have been proven.

Therefore, the proposed transceiver offers advantages regarding low manufacturing cost, good flexibility, and multifunctionality, since no correlation functions have to be computed, and no extra radio frequency, antenna, and wave beam are needed.

\section{Conflict of Interests}

The authors declare no conflict of interests.

\section{Acknowledgment}

This work was supported by the National Natural Science Foundation of China (61271204).

\section{References}

[1] S. D. Blunt, P. Yatham, and J. Stiles, "Intrapulse radar-embedded communications," IEEE Transactions on Aerospace and Electronic Systems, vol. 46, no. 3, pp. 1185-1200, 2010.

[2] D. Garmatyuk, J. Schuerger, Y. T. Morton, K. Binns, M. Durbin, and J. Kimani, "Feasibility study of a multi-carrier dual-use imaging radar and communication system," in Proceedings of the 4th European Radar Conference (EURAD '07), pp. 194-197, October 2007.

[3] C. Sturm and W. Wiesbeck, "Waveform design and signal processing aspects for fusion of wireless communications and radar sensing," Proceedings of the IEEE, vol. 99, no. 7, pp. 12361259, 2011.

[4] G. C. Tavik, C. L. Hilterbrick, J. B. Evins et al., "The advanced multifunction RF concept," IEEE Transactions on Microwave Theory and Techniques, vol. 53, no. 3, pp. 1009-1019, 2005.

[5] P. K. Hughes and J. Y. Choe, "Overview of advanced multifunction RF system (AMRFS)," in Proceedings of the IEEE International Conference on Phased Array Systems and Technology, pp. 21-24, IEEE, Dana Point, Calif, USA, May 2000.

[6] L. Xiao-bai, Y. Rui-juanand, and C. Wei, "Integrated radar and communication based on multicarrier frequency modulation chirp signal," Journal of Electronics and Information Technology, vol. 35, no. 2, pp. 406-412, 2013.

[7] L. Xiao-Bai, Y. Rui-Juan, and C. Wei, "The application of Poly-phase pseudorandom sequence in integrated radar and communication," Signal Processing, vol. 28, no. 11, pp. 1543-1550, 2012.

[8] M. Roberten and E. R. Brown, "Integrated radar and communications based on chirped spread-spectrum techniques," in Proceedings of the IEEE MTT-S International Microwave Symposium Digest, pp. 611-614, IEEE, Philadelphia, Pa, USA, June 2003.

[9] J. Song and Y. Liu, “The recognition of hybrid modulation signal combined with PRBC and LFM," in Proceedings of the 11th
International Conference on Signal Processing (ICSP '12), vol. 3, pp. 1720-1723, Beijing, China, October 2012.

[10] L. Wu and M. Feng, "On BER performance of EBPSK-MODEM in AWGN channel," Sensors, vol. 10, no. 4, pp. 3824-3834, 2010.

[11] Y. Yu and W. Lenan, "Studies of a dual-frequency EBSPKMODEM based radar-communication transceiver," Journal of Electronics and Information Technology, vol. 36, no. 8, pp. 17861791, 2014.

[12] M. Feng, L. Wu, and P. Gao, "From special analogous crystal filters to digital impacting filters," Digital Signal Processing, vol. 22, no. 4, pp. 690-696, 2012.

[13] M. Feng, L. Wu, J. Ding, and C. Qi, "BER analysis and verification of EBPSK system in AWGN channel," IEICE Transactions on Communications, vol. 94, no. 3, pp. 806-809, 2011.

[14] J. Sun, W. Fang, and W. Xu, "A quantum-behaved particle swarm optimization with diversity-guided mutation for the design of two-dimensional IIR digital filters," IEEE Transactions on Circuits and Systems II: Express Briefs, vol. 57, no. 2, pp. 141-145, 2010.

[15] Y. Yao, L. Wu, and J. Wang, "Composite broadcasting and ranging via a satellite dual-frequency MPPSK system," Mathematical Problems in Engineering, vol. 2013, Article ID 404357, 8 pages, 2013. 

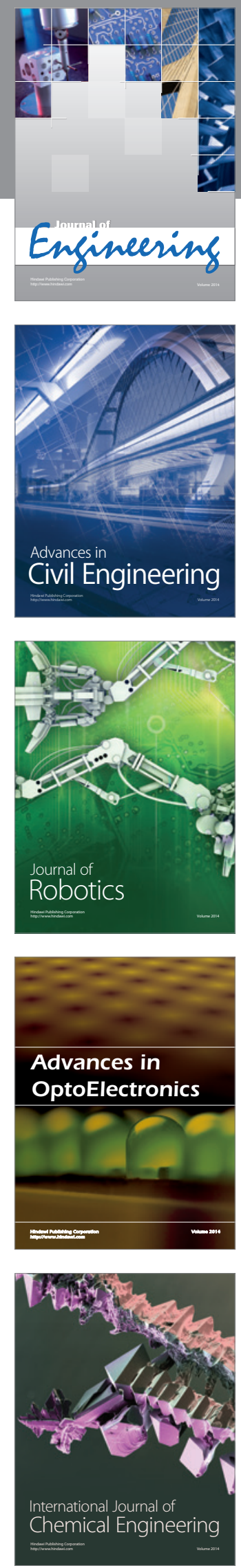

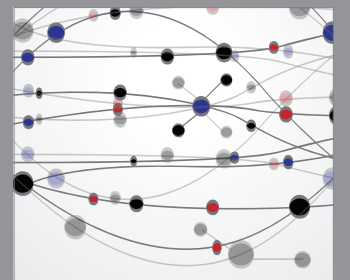

The Scientific World Journal
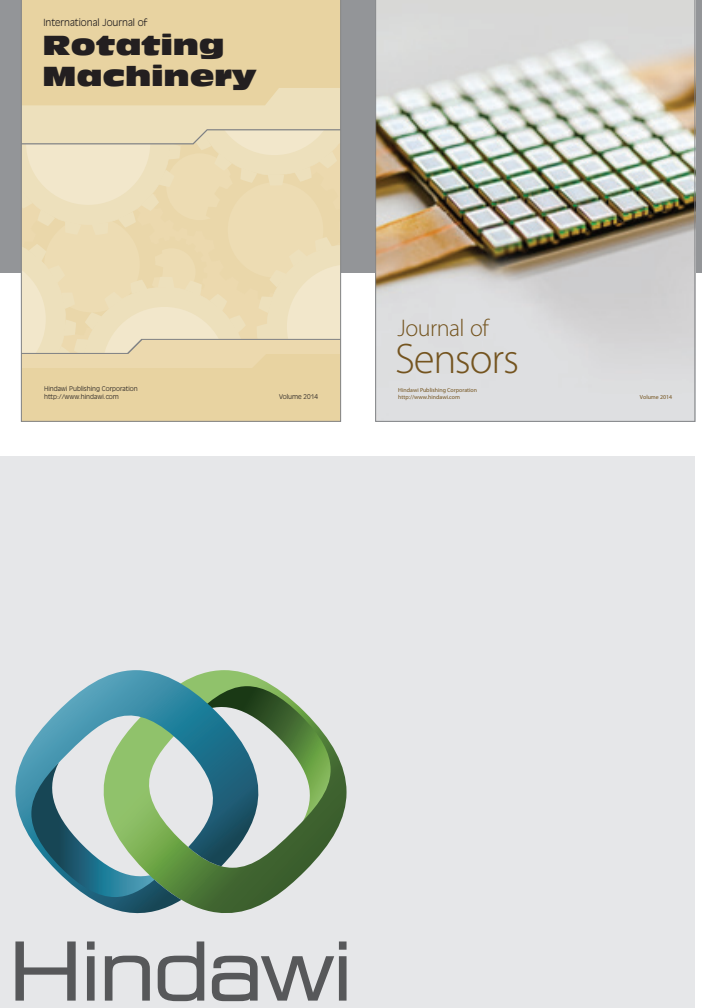

Submit your manuscripts at http://www.hindawi.com
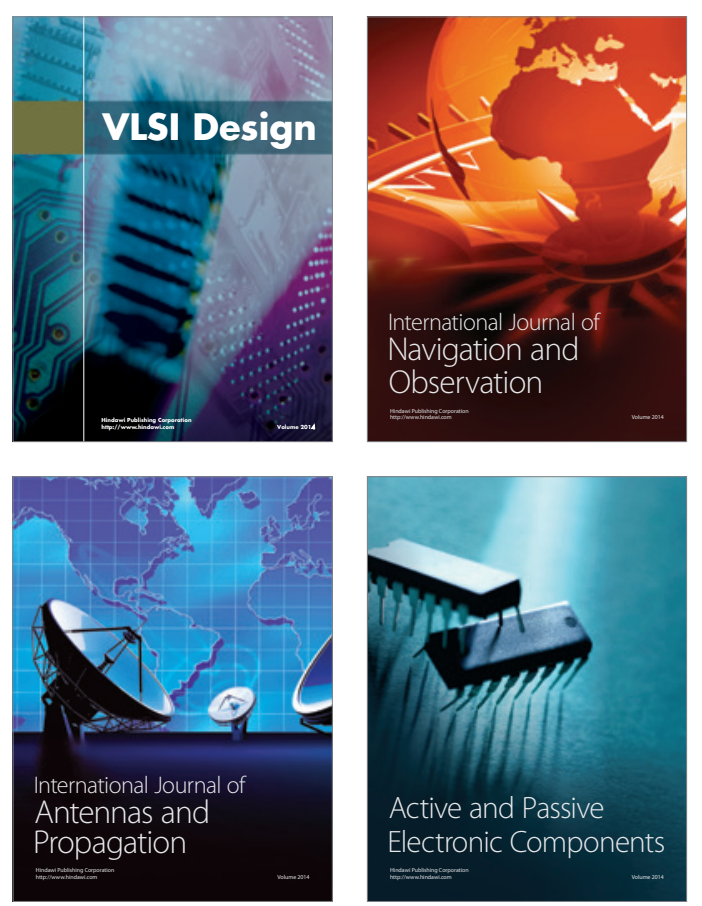
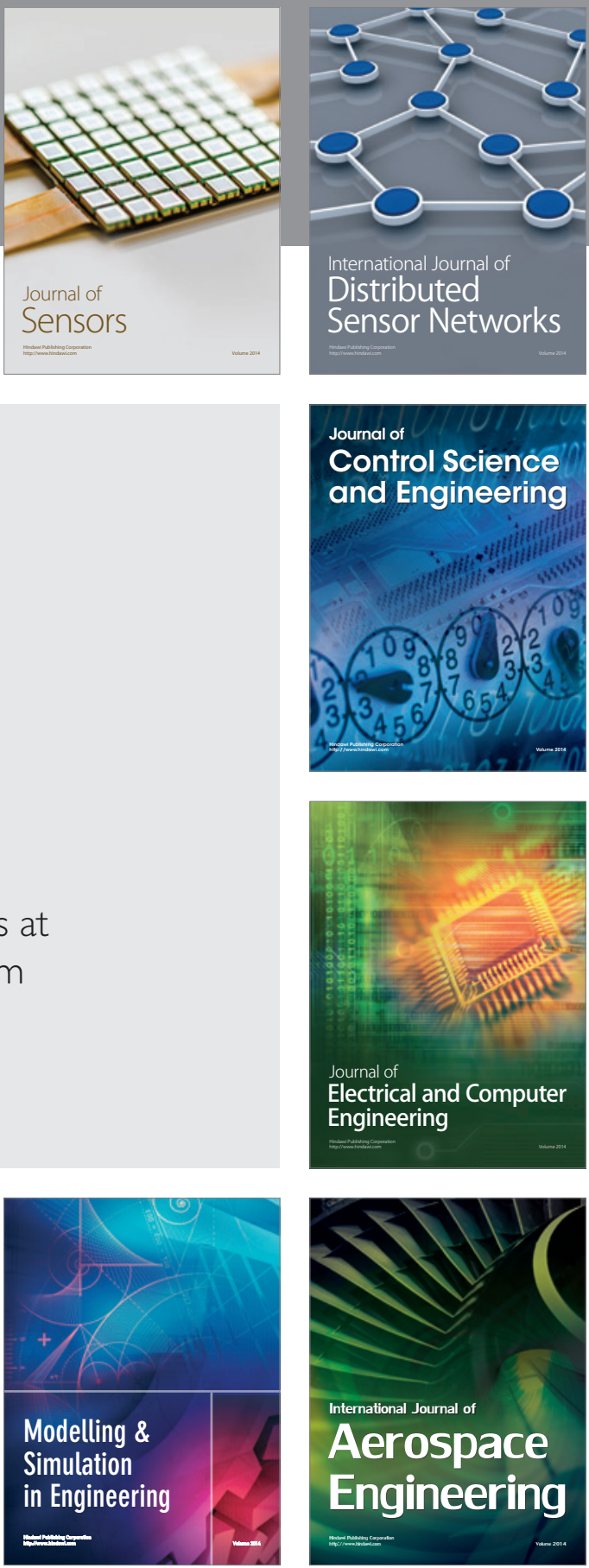

Journal of

Control Science

and Engineering
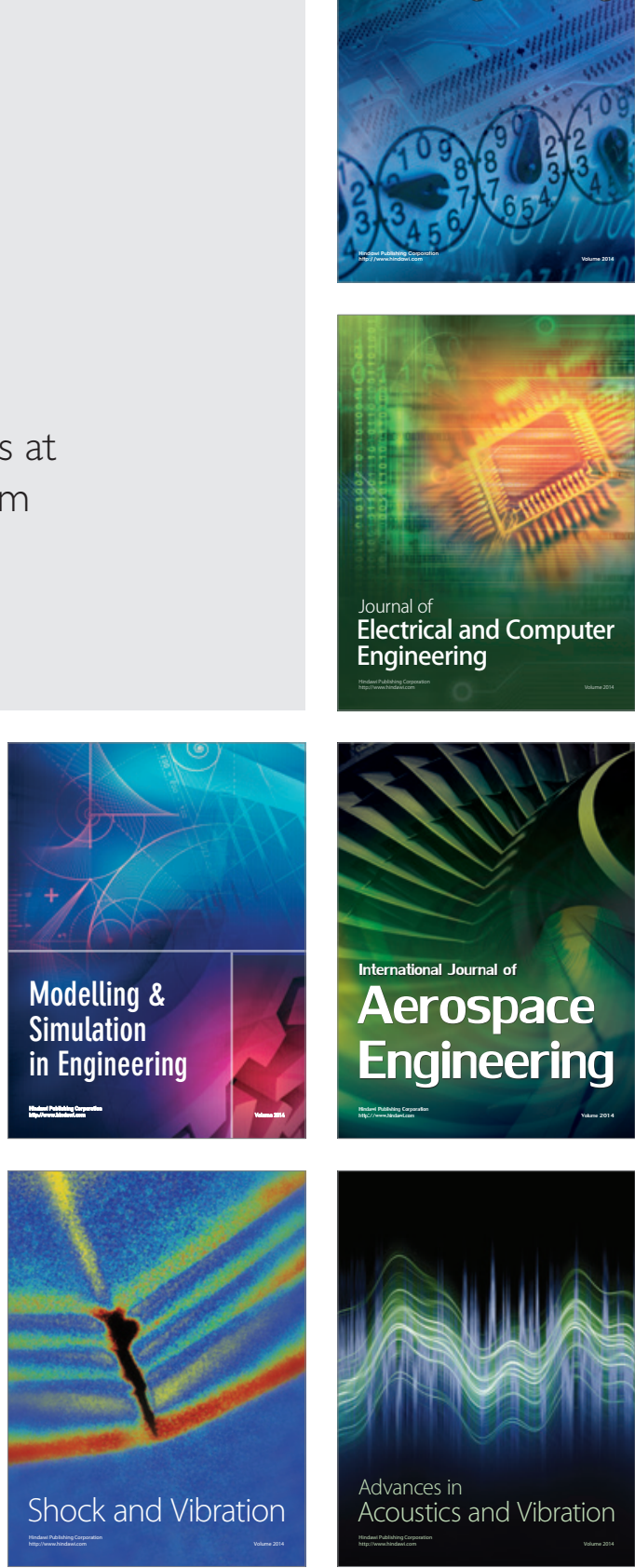